\title{
Modeling and Simulation Research on Accumulator effect of the Hydraulic Power Take-off System for a Wave Energy converter
} \author{
Jianjun Peng ${ }^{1,2, a}$, Yanjun Liu ${ }^{3, b, *}$ and Liping $\mathrm{Xu}^{1,2, \mathrm{c}}$ \\ ${ }^{1}$ College of Mechatronic Engineering, Henan University of science and Technology, Xiyuan Street, \\ Luoyang, China \\ ${ }^{2}$ Collaborative Innovation Center of Machinery Equipment Advanced Manufacturing of Henan \\ Province, Main University, Xiyuan Street, Luoyang, China \\ ${ }^{3}$ College of Mechanical Engineering, Shandong University,Jingshi Street, Jinan, China, \\ apjjsdu@163.com, ${ }^{\mathrm{b}}$ lyj111ky@163.com, ${ }^{\mathrm{c}}$ xlpzz@163.com \\ *corresponding author
}

Keywords: Wave energy; Modelling; Hydraulics; Gas accumulator; Wave Energy converter

\begin{abstract}
The latest research progress of the floating hydraulic wave power generating device -(Dragon I) is introduced in this paper. Dragon-I is a kind of point absorbing wave energy conversion device which uses hydraulic electricity generated system with energy storage function as the intermediate link to achieve the conversion of mechanical energy, hydraulic energy and electric energy. The mathematical model of the hydraulic power generation system is established, and the regulating function of the accumulator is analyzed in this paper. The simulation results show that the accumulator is obvious to the pressure and flow control and regulation in the hydraulic electricity generated system, which verified that the accumulator can stabilize the electric energy generated by the periodic motion excitation.
\end{abstract}

\section{Introduction}

The sea waves are a kind of huge energy source that has not been developed for the most, so it is very necessary to extract energy from the waves[1-3]. Patents on the use of ocean waves can be traced back to eighteenth century, while the modern study began in the oil crisis in 1870s. With the global warming and the increasing of carbon dioxide, the use of renewable energy to generate electricity has become a research hotspot. Point absorption is one of the power conversion devices that uses the sea waves to generate electricity, it uses hydraulic, linear motor, magnetic fluid motor or mechanical energy conversion system as the intermediate link of energy conversion to translate the wave energy into mechanical energy and then generate electricity. One type of energy conversion system based on hydraulic technology is widely used in the existing wave energy power generation devices, due to its several advantages such as wide range of stepless speed regulation, easy control, simple structure, small inertia and low cost[4-7]. However, it still exists some problems such as unstable energy output and low conversion efficiency[8-10]. In this paper, a kind of point absorption electricity generated device is proposed which makes use of the working principle of hydraulic energy storage, and it can greatly improve the stability of energy output.

\section{Mechanical model}

\subsection{The Hydrodynamics of Dragon I}

As shown in Figure 1 is the Dragon I. The buoy heaves along the vertical direction ( $x$ direction, horizontal plane: $x=0$ ), when it is considered as a simple single-degree-of-freedom model, as shown in Figure 2. The control equation of the body oscillations is

$$
m \ddot{x}=f_{h}(t)+f_{m}(t)
$$




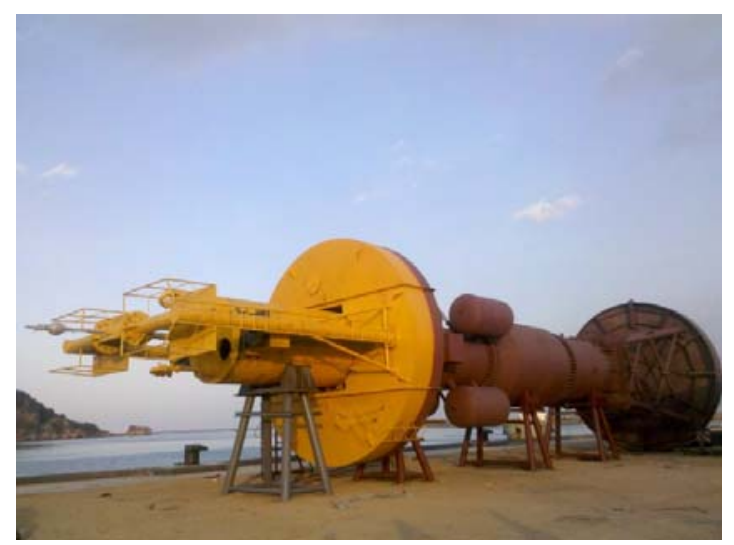

Figure 1 The prototype of the Dragon I

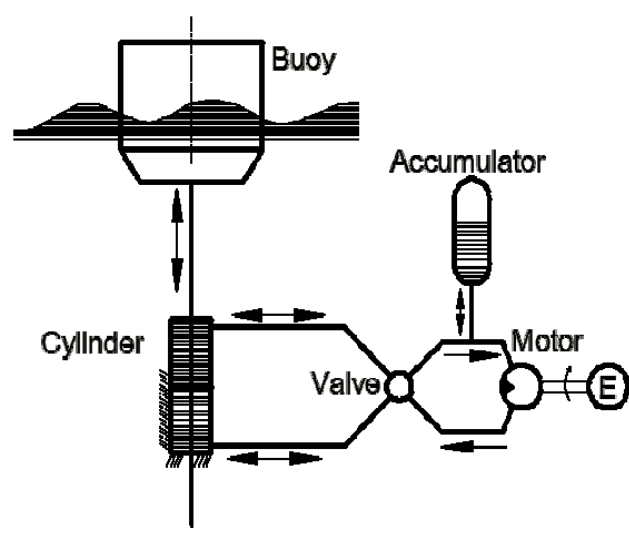

Figure 2 Schematic representation of the Dragon I

In which, $\mathrm{m}$ is the weight of the buoy, $\ddot{x}$ is the acceleration, $f_{h}$ is the wave force, $f_{m}$ is the force used to generate electricity (the PTO force) [5]. If the amplitude (the wave and the buoy) meets the requirements of the linear system, usually the wave force can be seen as follows

$$
\left\{\begin{array}{l}
f_{h}=f_{d}+f_{r}+f_{h s} \\
f_{m}=-K x-C \dot{x} \\
f_{h s}=-\rho g S x
\end{array}\right.
$$

In which, $\mathrm{f}_{\mathrm{d}}$ is the diffraction force, $\mathrm{f}_{\mathrm{r}}$ is the radial force, $\mathrm{f}_{\mathrm{hs}}$ the hydrostatic pressure. In a linear system, $-K x$ represents the action of the spring and $-C x$ represents the damping which is associated with the energy absorption ( $K$ and $C$ is constant). Where $\rho$ is the density of the sea water, $g$ is the gravitational acceleration and $S$ is the cross-sectional area of the buoy in static water.

When the incident wave is regular, the related displacement and force are sinusoidal functions of time $t$, and they can be expressed as[11]:

$$
\left\{\begin{array}{l}
x(t)=\operatorname{Re}\left(X_{0} e^{i \omega t}\right) \\
f_{d}(t)=\operatorname{Re}\left(F_{d} e^{i \omega t}\right)
\end{array}\right.
$$

In which, $X_{0}, F_{d}$ are complex amplitude, $\operatorname{Re}()$ is the real part.

Since the system is linear, $F_{d}$ and radial force $f_{r}$ can be expressed as:

$$
\left\{\begin{array}{l}
\left|F_{d}\right|=\left(\frac{2 g^{3} B(\omega)}{\omega^{3}}\right) A(\omega) \\
f_{r}(t)=-A(\omega) \ddot{x}-B(\omega) \dot{x}
\end{array}\right.
$$

Where, $A(\omega)$ is the added mass coefficient, $B(\omega)$ is the damping coefficient of the radiation. They can be calculated by commercial software based on the literature $[11,12]$.

Under the condition of linear system, the control equation of the body oscillations is

$$
(m+A) \ddot{x}+(B+C) \dot{x}+(\rho g S+K) x=F_{d} e^{i \omega t}
$$

We can take Eq.3 into Eq.8:

$$
X_{0}=\frac{F_{d}}{-\omega^{2}(m+A)+i \omega(B+C)+\rho g S+K}
$$




\subsection{Hydraulic System of Wave energy converter}

As shown in Figure 2 and Figure 3, most of wave energy conversion devices adopt the hydraulic cylinder as the power output device, and the relative movement between the hydraulic cylinder piston and cylinder generates the high pressure oil, which flows out from the left side through two group of the one-way valve. And the oil is absorbed from the tank trough the one-way valve on the right. The generator 12 works with the hydraulic motor and generates electricity. When the cylinder rod moves upward relative to the cylinder, the working process is contrary to the former, the down cavity absorbs oil through the one-way valve 2 and discharges, the down cavity discharge oil through the one-way valve 3 . The oil drives hydraulic motor 10 , and turns the generator 12 to produce electricity.

Denote by $p_{1}, p_{2}, p_{\text {up }}$ and $p_{\text {down }}$ the pressure of the high side and low side of the motor, the up side and down side of the cylinder, respectively. Assume that the process is a one-dimensional flow, and regardless of the upward or downward movement of the piston, it can be expressed by the following general formula :

$$
\left\{\begin{array}{l}
p_{1}-p_{2}=\left|p_{u p}-p_{\text {down }}\right|-k q^{2}-I \frac{d q}{d t} \\
q=S_{C} \frac{d x}{d t}
\end{array}\right.
$$

In which, $S_{C}$ refers to the effective area of the hydraulic cylinder and $x$ is the displacement of the piston. Hydraulic motor is driven by the pressure difference between the both side, $p_{1}(t)-p_{2}(t)$, changing over time. Simplify the process by ignoring the liquid inertia and pipe pressure loss of hydraulic circuits then we got the Eq.7 $k=I=0$. Therefore, $p_{1}-p_{2}=\left|p_{u p}-p_{\text {down }}\right|$ when the piston moves.

When the floating body moves, control equation would refer to Eq. 1. Sometimes, the velocity of the floating body, changing over time, will be zero. At this time, the floating body remains still until the hydrodynamic force act on the floating body $f_{d}(t)-\rho g S x(t)-\int_{-\infty}^{t} L(t-\tau) \ddot{x}(t) d(\tau)$, overcomes the resisting force $\Phi=S_{C}\left(p_{1}-p_{2}\right)$. Then the hydraulic oil can be pumped to the accumulator. The gas accumulator is used to "peak clipping fill valley", so the motor can move smoothly.

The instantaneous power and the time-average power of the wave energy converter can be represented as:

$$
\left\{\begin{array}{l}
P(t)=\Phi|\dot{x}(t)| \\
\bar{P}=\int_{t_{0}}^{t_{f}} P(t) d(t) / \Delta t
\end{array}\right.
$$

In which, The value of $\bar{P}$ depended on the time interval $\Delta t=t_{f}-t_{0}$. Practically, in order to ensure error on $\pm 1 \%, \Delta t$ must be less than fifteen minutes. 


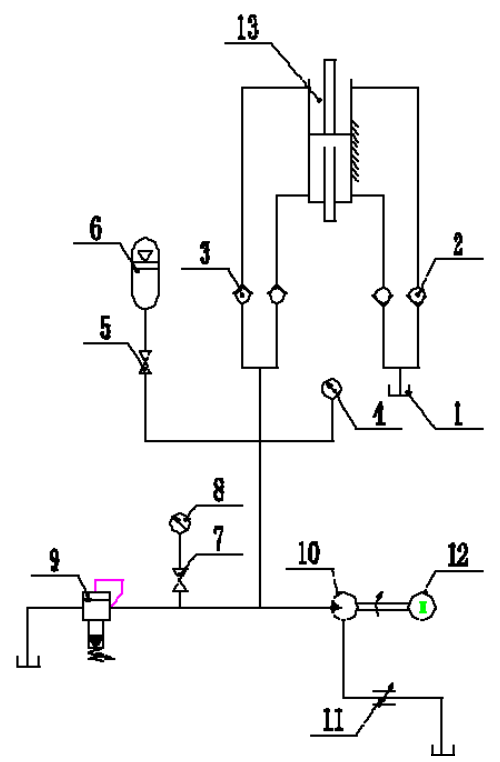

1-hydraulic oil container; 2- oil-absorbing one-way valve; 3-oil outlet one-way valve ; 4-pressure gage; 5globe valve; 6-energyaccumulator; 7-isolating valve; 8-pressure gage; 9-safety valve; 10-hydraulic motor;

11-throttle value; 12-generator; 13-hydraulic cylinder

Figure 3 Schematic representation of the hydraulic system

\subsection{Mathematical model of the gas accumulator}

The role of the accumulator 6 makes the power system work steadily and also improves the rate of the energy utilization, when the high pressure oil comes out from the hydraulic cylinder, the accumulator can store part of the energy, and also reduce the impact on the power system from the instantaneous high pressure; In the same way, when the output of the hydraulic cylinder is less and the pressure of the oil is smaller, the accumulator can release some energy and maintain power systems working properly. In order to analyse the effect of accumulator more accurately, the mathematical model of accumulator is established as shown in Figure 4.

In the actual analysis process, we divided the accumulator into two parts: the gas chamber and the liquid chamber and neglected the influence of the connecting line. We did the stress analysis of the join points-oil chamber between the accumulator and the hydraulic circuit to get the mechanic model of the accumulator. Then according to the relationship of the pressure and flow between different parts, the full mathematical model of the accumulator was got by analysing the mechanic model $[13,14]$.

Assumptions are as follows: the oil compressibility could be ignored and flows in the accumulator could be considered as laminar flow, the time-average temperature of the gas in the accumulator could keep same with the surrounding in the different sea conditions and so that it is an isothermal process.

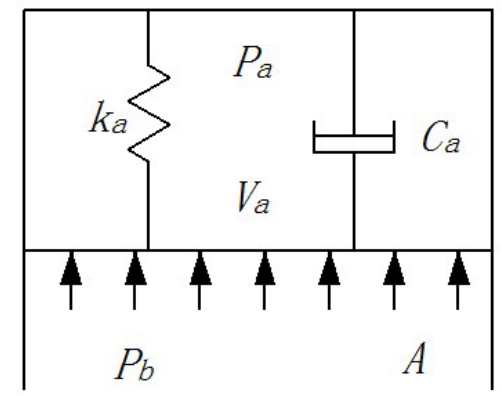

Figure 4 The model of accumulator

Based on the analyses of the system the chamber stress model of the accumulator is: 


$$
\left(p_{b}-p_{a}\right) A=k_{a} \frac{V_{a}}{A}+C_{a} \frac{\dot{V}_{a}}{A}
$$

Where, $p_{b}$ is the oil pressure of the accumulator, $k_{a}$ is the gas stiffness coefficient of the skin at any time and $C_{a}$ is the damping coefficient of the gas. Set $q$ for the inlet flow of the accumulator, which implies that

$$
q=-\dot{V}_{a}
$$

We take Eq.10 into the formula Eq.9 and then Laplace transform as shown in:

$$
\left[p_{b}(s)-p_{a}(s)\right] A=-\left(\frac{k_{a}}{A \cdot s}+\frac{C_{a}}{A}\right) q(s)
$$

When the impact of the connecting line and the elastic modulus of the oil is neglected, we can get the mathematical model for the output of the accumulator's chamber volume after the Laplace transform

$$
G(s)=\frac{V_{a}(s)}{p(s)}=\frac{A_{a}^{2}}{m_{a} s^{2}+\left(B_{b}+C_{a}\right) s+\left(k_{a}+\frac{k A_{a}^{2} p_{a 0}}{V_{a 0}}\right)}
$$

Where, $A_{a}$ is the accumulator's cross section (Assumed that the area at the bottom of the bag is equal, $\left.A=A_{a}\right), m_{a}$ is the quality of the oil cavity, $p_{a 0}$ and $V_{a 0}$ respectively indicates as the initial air pressure and the volume of the accumulator, $B_{b}$ is approximate to the oil cavity damping coefficient.

It can be seen from the Eq.12 that when the impact of the connecting line is neglected the performance of the accumulator is mainly about the oil damping, the air volume, the cross-section area and the air pressure.

\section{Numerical Simulations}

According to the mathematical model shown in Eq. 9 and Eq.12, a numerical simulation study has been completed for the incident response of accumulator under the impact of pressure. Using the parameters shown in Table 1, to set the pneumatic pressure of the accumulator, $p_{a 0}=6 M p a$. The amplitude of input step signal was 1 which represented that the input pressure pulsation was 1Mpa. The simulation results shown in Figure 5, after a short vibration, the accumulator volume reached a steady state with time $2.5 \mathrm{~s}$. The response time meet the requirements.

In order to verify the regulatory role of the accumulator to the hydraulic system, a simulation model of the hydraulic power system (as shown in Figure 3) was established. The input displacement signal of the hydraulic cylinder as shown in Figure 6, which represents the wave motion of the floating body. The initial position of the hydraulic cylinder piston was $1 \mathrm{~m}$, which means the distance from the piston to the bottom of the hydraulic cylinder. Then the piston moved upwards, and set the simulation stroke is $1.2 \mathrm{~m}$ (the total stroke of the hydraulic cylinder $4 \mathrm{~m}$ ), reciprocating motion twice, and then stop. The generator connected to the motor which was collected pressure at the inlet of the hydraulic motor, speed, torque, flow rate at the entrance, the motor output power, as shown in Figure 7, Figure 8, Figure 9, Figure 10, Figure 11.

From the simulation results shown in above, the output pressure was to maintain basic stability. The tendency of pressure, flow, speed, torque were consistent. When the hydraulic cylinder motion stopped, the pressure reaches the maximum (85bar). And then the generation continued about 40s because of the role of the accumulator. 


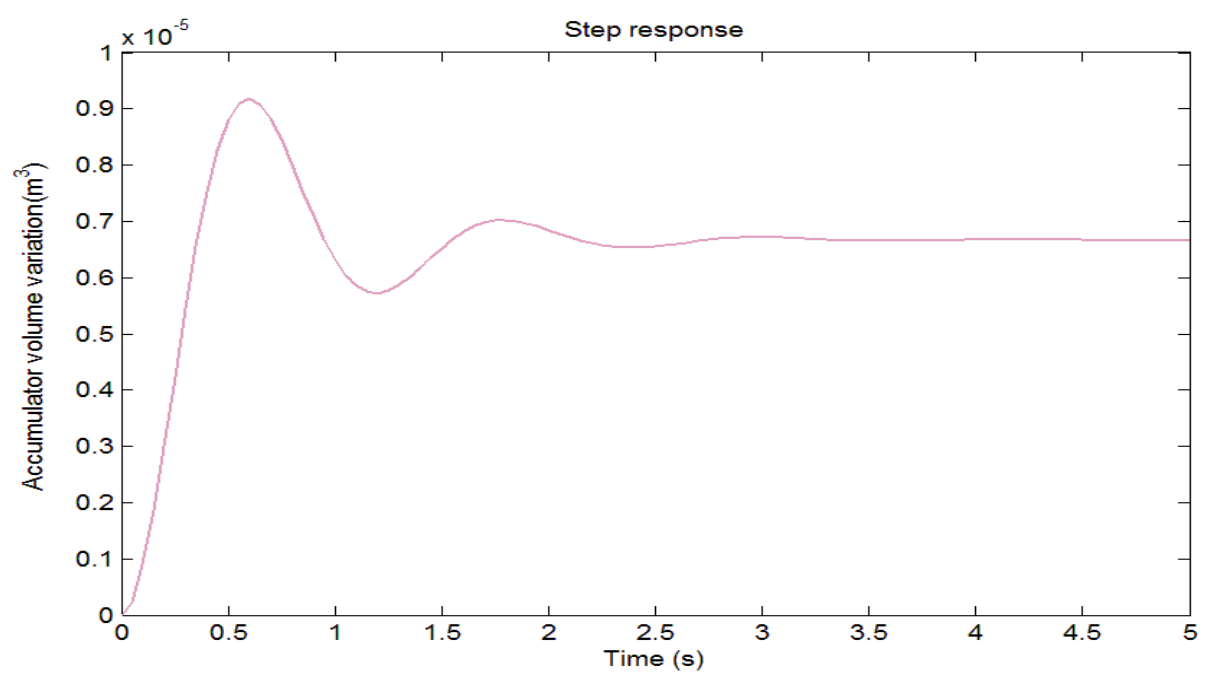

Figure 5 The time response of the accumulator under the pressure shock.
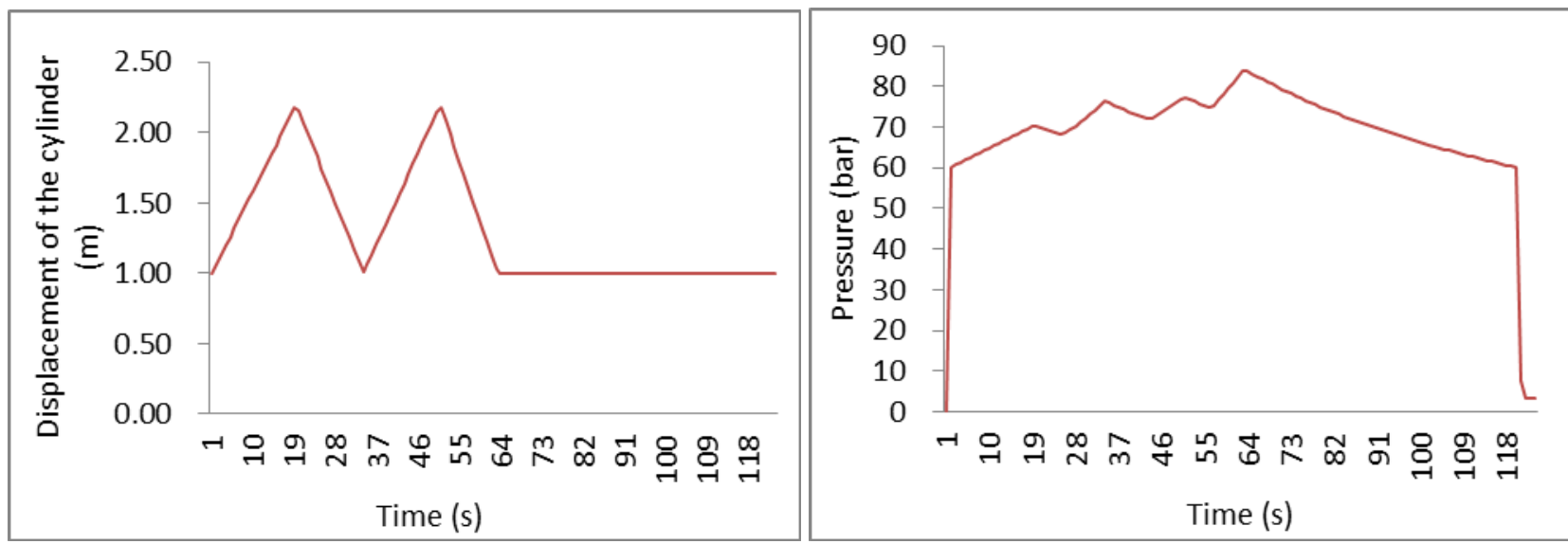

Figure 6 The displacement signal of the hydraulic cylinder

Figure 7 The pressure of the hydraulic motor

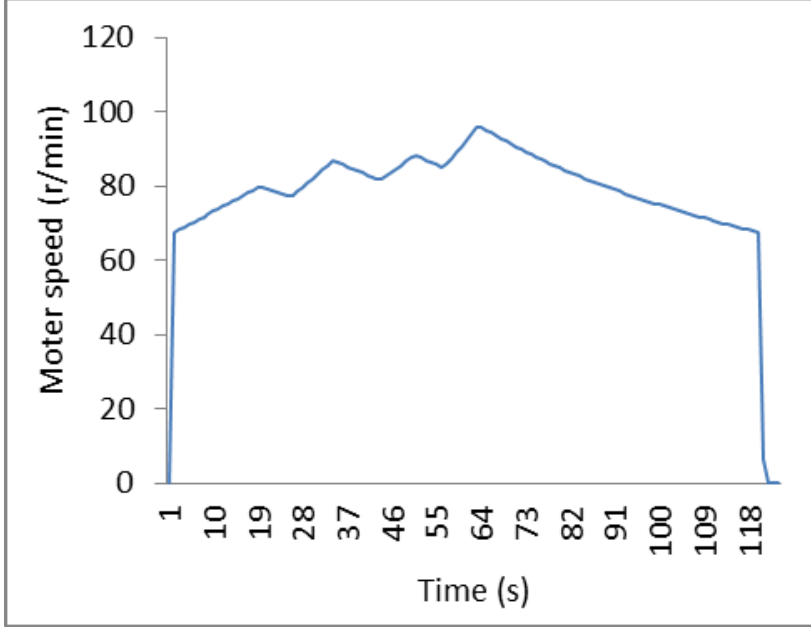

Figure 8 The revolving speed of the hydraulic motor

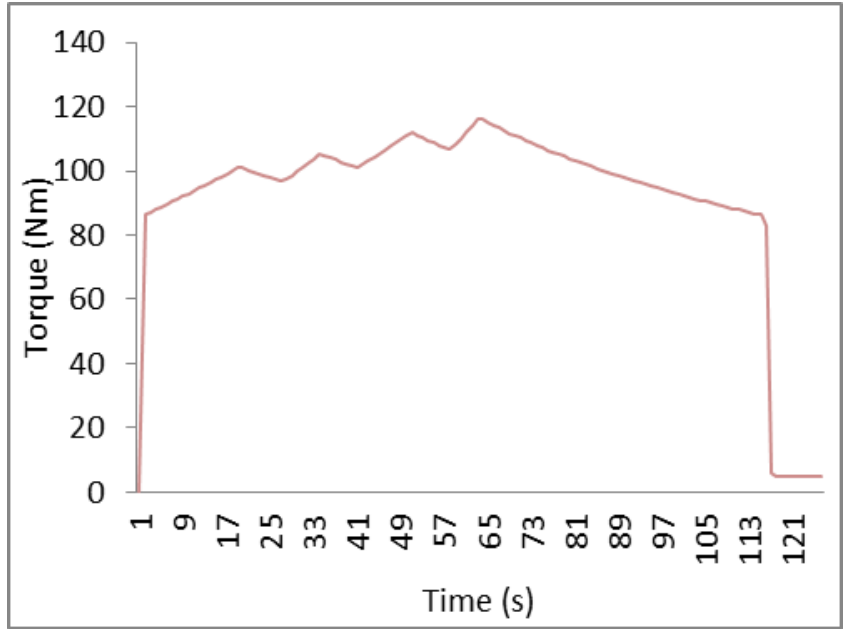

Figure 9The torque of the hydraulic motor 


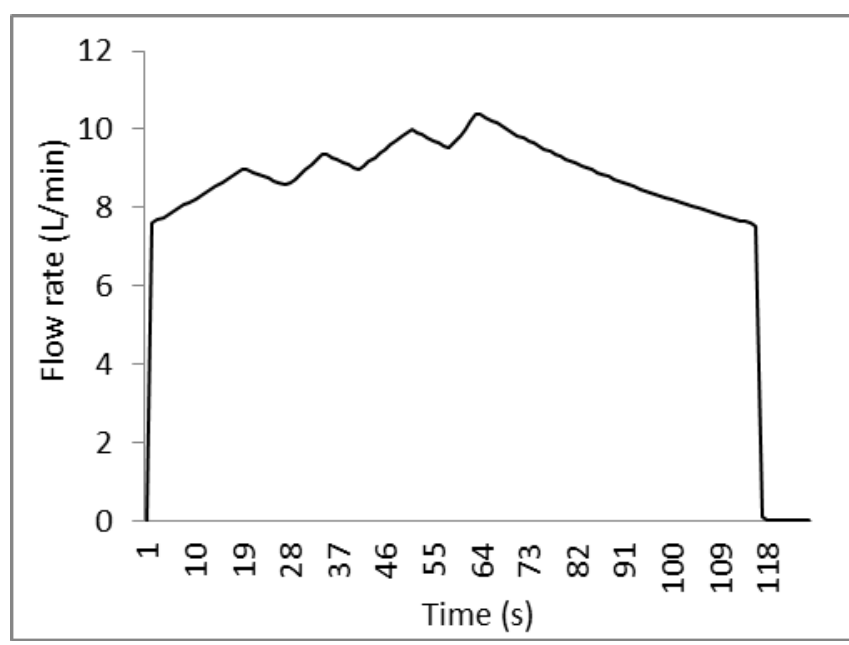

Figure 10 The flow rate of the hydraulic motor

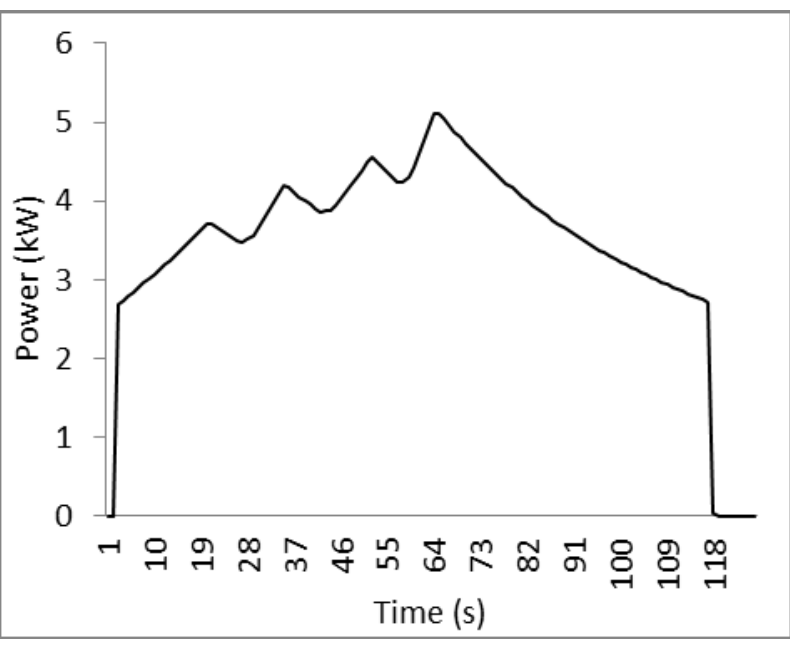

Figure 11The output power of the hydraulic motor

Table1 Simulations parameters

\begin{tabular}{|c|c|}
\hline Name & Parameter \\
\hline Stroke of Hydraulic Cylinder (m) & 1 \\
\hline Diameter of piston rod (mm) & 130 \\
\hline Diameter of cylinder (mm) & 160 \\
\hline Accumulator capacity (L) & 40 \\
\hline Accumulator pressure (Mpa) & 6 \\
\hline Gear motor displacement (ml/r) & 100 \\
\hline Nominal pressure/Max (Mpa) & $12.5 / 20$ \\
\hline Motor rated speed/(range) (r/min) & $2000 / 150 ~ 2500$ \\
\hline Three-phase alternator rated speed(r/min) & 1500 \\
\hline Nominal voltage $(\mathrm{V})$ & 380 \\
\hline Rated power $(\mathrm{kW})$ & 35 \\
\hline
\end{tabular}

\section{Conclusion}

This paper makes a brief introduction to the floating-point wave power generation device and establishes a mathematical model of single degree of freedom under the linear wave conditions. This paper also analyses the hydraulic system of the device and establishes a mathematical model for the accumulator. We also complete a series of numerical simulations, which indicates that the accumulator makes significant differences to the adjustment of the pressure of the system. The gas accumulator is very important for the whole wave energy conversion device. On one hand, it is limited to the maximum load, pressure, available space and equipment cost; On the other hand, the design of the accumulator will affect the both sides of the energy conversion chain: the dynamic performance of absorbing water and the performance of the electrical equipment, the relationship of the both side has been established, which provides design basis for the designers.

It should be noted that linear wave theory is the basis of the hydrodynamic theory, with which we can extract energy from waves in this paper. But the linear wave theory does not include the practical situations of large vibration of floating body, wave of high size and the actual fluid viscous dissipation, which will be dealt with in effective ways in the future.

\section{Acknowledgements}

This work was supported by the Renewable Energy Special Fund of the State Oceanic Administration of China (No. GHME2010ZC01), by the National Science Foundation for 
Young Scientists of Henan University of Science and Technology ( No. 2015QN017), and by the Program for Innovative Research Team of Henan University of Science and Technology (No. 2015XTD012 ). Meanwhile, the authors would like to thank Professor Yanjun Liu for his detailed guidance and his collaborators for their great help.

\section{References}

[1] Ross, D. 1995. Power from the waves, Oxford University Press, Oxford, UK. Salter. S. H, 1974. "Wave power," Nature, 249(5459), 720-724.

[2] Verbrugghe, T., Kortenhaus, A., \& Rouck, J. D. 2015. Numerical modelling of control strategies and accumulator effect of a hydraulic power take-off system. Oceans,1-10, IEEE.

[3] B. Drew, A R Plummer and MN Sahinkaya. 2009. "A review of wave energy converter technology", Proc. IMechE , Part A: J. Power and Energy 223, 887-888.

[4] A.F.O. Falcão, 2010. Wave energy utilization: a review of the technologies, Renewable and Sustainable Energy Reviews 14, 899-918.

[5] Fan, Y. J., Mu, A. L., \& Ma, T. 2016. Design and control of a point absorber wave energy converter with an open loop hydraulic transmission. Energy Conversion \& Management, 121, 13-21.

[6] Henderson, R., 2006. Design, simulation and testing of a novel hydraulic power take off system for the Pelamis wave energy converter. Renewable Energy 31,271-283.

[7] Josset, C., Babarit, A., Clement, A.H., 2007. A wave-to-wire model for the SEAREV wave energy converter. Proceedings of the Institution of Mechanical Engineers, Part M-Journal of Engineering for the Maritime Environment 221 (2), 81-93.

[8] Sheng, W., \& Lewis, A. 2016. Power take off optimization for maximizing energy conversion of waveactivated bodies. IEEE Journal of Oceanic Engineering, 41(3), 1-12.

[9] Limin Yang, Torgeir Moan, 2011. Dynamic analysis of wave energy converter by incorporating the effect of hydraulic transmission lines., Ocean Engineering 38, 1849-1860

[10] Aurelien Babarit, Michel Guglielmi, Alain H. Clement, 2009. Declutching control of a wave energy converter. Ocean Engineering,36, 1015-1024.

[11] A.F.O. Falcão, 2002. Control of an oscillating water column wave power plant for maximum energy production, Applied Ocean Research,24, 73-82.

[12] A.F.O. Falcão, 2007. Modelling and control of oscillating-body wave energy converters with hydraulic power take-off and gas accumulator, Ocean Engineering,34, 2021-2032.

[13] Yang Byung-Do, Kim lee-Sup, 2002. A high speed direct digital frequency synthesizer using a low power pipelined Parallel accumulator on Circuits and Systems. IEEE International Symposium 5, 373-376.

[14] Quan Lingxiao, 2005. "Research on the bag accumulator's basic theory and experiment based on pipeline effect", M. Chinese. thesis, Yanshan University, China, March,85-93. 